\title{
Index of Topics, Motifs, Images, Places, and Devices
}

absence of God - see God

absurdity - see comedy

Adorno's statement - also see crisis of literature

in the face of war crimes $6,71,165-166$,

175, 180, 187, 382-383, 435, 478

aliyah - see postwar emigration for Palestine

allosemitism 130-131

anonymous death - see death

anthropomorphisation 173

anti-Jewish campaigning in the late 1960s, March

1968 8, 57, 67, 161, 203, 222, 233, 335

anti-Jewish skinhead riots 381

antisemitic behaviour of Czechs 16, 115, 134, 287, 356

antisemitic behaviour of Poles 58, 215, 222, 235, $271,301,307,330,344,359,374,394,408$, 422

antisemitic behaviour of Slovaks 19-20, 151,

304, 316, 377-378, 401-402, 404-455

antisemitic prejudice $293,297,308,325$

antisemitic stereotypes - see stereotypes in presentation of the Jews

apelplac 84, 196, 387

Arab-Israeli War 1948 149, 236, 348

Arab-Israeli War 1967 (Six-Day War) 17, 33, 111, 137, 149, 348

Arcadia 50, 293, 351

Armia Krajowa (Home Army) 106, 179, 330, 350, 392

Armia Ludowa (People's Army) 57, 129

Aryan papers (false identity) 3, 57, 73-74, 96, 218, 234, 263, 276, 358, 361, 380, 385, 393, 426, 478

aryanisation, seizure of Jewish property 19,96 , $152,211,304,386,401-402$

assimilated Jew - see Jew

Auschwitz, Auschwitz-Birkenau - see concentration camps

authorial narrator - see narration

author-narrator - see narration

autobiographical narrative - see narration

autocensorship - see censorship

banality of the Evil (Hannah Arendt) 54-55, 180, 282, 290

baptism of Jews - see conversion of Jews to Christianity beautiful Jewess - see stereotypes in presentation of the Jews

Betżec - see concentration camps

Będzin Ghetto - see ghettos

Bergen-Belsen - see concentration camps

Białystok Ghetto - see ghettos

Bible/Hebrew Bible/Old Testament 90, 100, 113, 242, 374, 381, 405, 408, 456, 458 - also see Torah

Book of Deuteronomy 93

Book of Ecclesiastes (Kohelet) 265, 458

Book of Esther 454

Book of Genesis 46

Book of Jeremiah 321

Book of Job 112, 123, 371, 439

Book of Judith 320

Book of Lamentations 419

Book of Psalms 138, 202, 296

Book of Ruth 106

Cain and Abel 405, 408

Noah's Ark 77, 381

Song of Songs 102, 103, 458

Tower of Babel 108

Bible/New Testament 108, 409

Book of Revelation 101, 451

Gospel of John 435

The Gospels 122, 283

Mary and Martha 409

blackmailer - see denouncement of Jews

blasphemy 250,444

body, body and soul - see corporeality

Buchenwald - see concentration camps

bystander

Czechs, Poles and Slovaks as bystanders in general 119

guilty bystander 104, 267-268, 275, 290, $312,370-371,312,377,393-394,405$

helping bystander $67,64,133-134,257$, $276,345,355,405$

Cain and Abel - see Bible/Hebrew Bible/Old

Testament

carousel at Krasiński Square in Warsaw 371, 436

Catholicism, Catholic priest 17, 19, 26, 57-58, 96-97, 150, 169, 182, 200-202, 211, 214, 227, 234-235, 243, 264, 271, 315, 381, 385, $390,401,405,431$ 
censorship $8,10,11,24,25,32,74,97,102,107$, $125,134,143,220,234,249,263,268,307$, 318-319, 358, 407

autocensorship 307

change of identity - see identity of Jews change of name - see names of Jews

Charter 77 95, 102, 136, 253, 266, 278, 325

Chełmno - see concentration camps

child's narration, child's point of view see narration

child's narration \& adult's comments see narration

children

lost childhood $62,89,141,145,148,230-$ 231, 235, 304-305

children as victims 103

circumcision 234

circus (persecution of Jews compared to a circus) 86,257

clerofascist regime in Slovakia $17,19,26,27,66$, $96,151,169,211-212,263,265,394-396$, 400-402, 404, 453, 458

collaboration of Jewish councils with the Nazis see Judenrat

collaborator - see denouncement of Jews

collage (montage) 21, 71, 158, 266, 326, 373

collective memory - see memory

comedy - also see humour, irony, satire absurdity $108,117-118,130,131,191,211$, $249,252,279,296,297,347,389,412$, 450

grotesque $3,18,19,23,28,32,47,54,55$, 97, 108, 117, 136, 166-167, 191, 211, $226,259,279,287,293,297,300,322$, $326,382,386,389,415,417,428$

commemoration of the Holocaust $6,15,30,76$, $137,149,162-163,173,176,186-187,261$, $289,347,352,412-413,432$

communicative memory - see memory communist $67,85-86,96,100,134,165,205$, $226,254,257,296,355,381,385,410,447,449$

Communist resistance - see resistance composition

anticipation \& foreshadowing $60,166,297$, 446

cycle 276

flashbacks 73, 76, 111, 126, 127, 147, 148 , $158,166,208,237,286,316,355$, 380-381, 396-397, 404 fragmentation $61-62,72,73,137,276-277$, 326, 409

frame narrative, background story 126,158 , $169,191,274-276,355,396$

prospective into the future lives 58, 307 concentration camps

Auschwitz, Auschwitz-Birkenau 6, 13, 15, $19,21,25,26,28,29,41,42,43,45$, $84,85,86,99,104,118,119,126,132$, $136,139,141,143,144,145,147-148$, 154-156, 157, 158, 172, 173, 179-180, 182, 183-184, 191, 194-195, 200-201, 207-208, 211, 212, 229, 221, 231, 237239, 241, 242-244, 245, 254, 256, 260, 261, 270, 272, 285, 256, 326, 327, 335, $338,339,342-343,365-367,380$, 388-389, 396-398, 404, 411-412, 414, 416, 418, 419, 423, 430, 453, 457 Family Camp in Auschwitz 158, 241, 242

Betżec 173, 394

Bergen-Belsen 397, 423, 452

Buchenwald 41, 42, 117-119, 166, 338, 418

Chełmno 190, 271

Dachau 84, 139, 143, 144, 182, 338, 416

death camps $31,137,151,186,238,254$, 271, 385, 414, 423, 427

fictitious concentration camp 281-283

Flossenbürg 172

Gleiwitz (Gliwice) 42

Gross-Rosen 423

Janowska 392

Kaufering 84, 85-86, 143, 144

Kurzbach 342

Majdanek 271, 408

Mauthausen 25, 165-167, 245

Mittelbau-Dora 423

Monowice 41, 42, 418

Natzweiler-Struthof 182

Oberlanzendorf 74

Pruszków 71

Ravensbrück 201-202, 271

Sachsenhausen 57, 143

Schwarzheide 241

Treblinka 25, 63, 89, 99, 106-107, 166, 172-174, 256, 317, 318, 352, 426-428, 442, 443-444

Treblinka trials in Düsseldorf 426 Treblinka Uprising 426 
confession narrative - see narration conversion of Jews to Christianity (baptism of Jews) 96-97, 121, 150, 218, 243, 260-261, 271, 304, 343, 385-386, 401, 447 corporeality, body, body and soul 20,131, 157$158,239,250-252,293,323$

crematorium 28, 45, 113-115, 173, 184, 327

crisis of literature in the face of war crimes 176 , 371, 392-393, 436 - also see Adorno's statement impossibility of expressing the Shoah 82, 162, 194-196, 323, 327, 351 cultural activities in Theresienstadt - see ghettos, Theresienstadt

cultural memory - see memory

cycle - see composition

cynicism 183-184, 282,289-290

Czech-German relations - see relations

Czech-Jewish relations - see relations

Czech-Jewish-German relations - see relations

Czech Jews-Polish Jews relations - see relations

Czech-German relations - see relations

Dachau - see concentration camps

Daniel - see names of Jews

David - see names of Jews

death

anonymous death 219

death as Death personified 62, 242, 258

death camps - see concentration camps death in gas chamber $32,41,42,145,167$, 184, 241, 242, 343, 366, 411, 418

dignified death 100, 158, 200-201, 366, 439, 444, 451

undignified death $63,183-184,243,444$, 450

death march 32, 238, 239, 241, 342

denouncement of Jews (collaborator, informer, blackmailer, shmaltsovnik) 58, 67, 72, 74, 114, 197, 216, 258, 319, 355, 393, 401, 402, 444

denying the Holocaust 448

deportation - see transport

Devil 137, 191, 242, 252 - also see Hell, Mephisto, Satan

diary - see narration, diaristic narration

dignified death - see death

docudrama 212, 417, 456

documents (historical facts) $5,13,21,43,72,151$, 186, 212, 234-235, 238, 261, 264, 271, 335,
$370,386,396,397,416,418-420,432-$

433, 435, 458

double identity - see identity

dream scenes, surreal scenes $9,23,55,108$,

165, 170, 204, 268, 287, 296-297, 301, 322,

331, 378

daydreams as an escape 46

drugi obieg 57, 358, 362 - also see samizdat

Düsseldorf trials - see concentration camps, Treblinka

dybbuk 347

Eichmann - see Nazi leaders and prominent Nazis

Elders of the Jews in Theresienstadt - see ghettos and Judenrat

Emanuel - see names of Jews

emigration

emigration from Czechoslovakia after the

Soviet invasion in August of 1968 23,

25, 104, 125, 139, 266, 338, 376, 384,

426

emigration in 1939 24, 110, 380, 381

emigration in 1948 and 1949 20, 58, 381, 110, 241

emigration in 1956 and 1957 7, 361

emigration to Argentina after 1945212 , 263, 388

emigration to Israel in or after 196823 , $53,58,125,139,143,338,376,378$, 381

emigration to Palestine after the war, aliyah 7, 20, 53, 110, 125, 209, 212, 226, 237-239, 241, 249, 361, 414, 446

emigration to the U.S. and Canada 23, 139, 203, 226, 233, 238-239, 263, 338, 378,397

Esther - see names of Jews

European identity - see identity

eyewitness - see witness

fact and fiction 3, 42-44, 61-64, 151-152, 172$174,183,190-191,212-213,234-235,238-$ 239, 261, 263-265, 270-271, 290, 386, 418-421

family camp in Auschwitz - see concentration camps, Auschwitz

family memory - see memory 
family novel, family history $12-14,24,27,84$, 187, 197-199, 230-231, 264, 347, 396-398, 430, 457-458

famine - see hunger

fear 10, 47, 61-63, 74-75, 184, 198, 204-205, 231, 239, 242, 258, 268, 296, 301, 303-305, 311, 330, 355-356, 393-394, 408, 451

female experience of the Shoah 13, 14, 147-148, 212, 195-196, 201-202, 208-209, 237-239, 315-316, 322-323, 329-332, 335-336, 340, 342-344, 355-356, 363, 397, 453-454 also see narration, femaleperspective

female identity - see identity

female perspective - see narration

fictitious concentration camp - see concentration camps

first-person narrator - see narration

Final Solution 103, 340, 386, 419

flashbacks - see composition

Flossenbürg - see concentration camps

fragmentation - see composition

frame narrative, frame story - see composition

free verse - see verse

Führer - see Nazi leaders nad prominent Nazis

gas chambers $26,41,42,89,118,131,139,144$, $167,184,208,241,242,296,318,338,343$, $344,365,366,380,411,416,418,457$

gender, gender stereotypes 255, 314, 316, 454 ghettos

Będzin Ghetto 143, 183

Biatystok Ghetto 344

Izbica Ghetto 73, 74

Lodz (Litzmannstadt) Ghetto 4, 19, 47, 92-94, 140, 190-192

Lviv Ghetto 198

Międzyrzec Podlaski Ghetto 271

Otwock Ghetto 12, 106-107, 439

Pruszków Ghetto 63

Sambor Ghetto 129

Theresienstadt Ghetto $18,19,21,25,28$, 29, 31, 41-44, 45-48, 84, 85, 86, 94, $99,104,132,134,136-138,139,157$, 159, 208, 241, 245-247, 253-255, 256, $258,275,285,338,342,344,355,356$, 381, 388-390, 396-398, 414-417, 418-421, 426, 427, 451, 457, 458

cultural activities $18,42,84,245,247$, 416, 418-421 elderly people in Theresienstadt 47 , 344

Elders of the Jews in Theresienstadt 42, 43, 47, 245, 416

filming in Theresienstadt 415 music in Theresienstadt 18, 418-421

Red Cross delegation 239, 416, 419, 421

sex in Theresienstadt - see sex

Warsaw Ghetto 4-5, 8, 63, 80-82, 88-90, 119, 186-188, 215, 271, 299-300, $317-318,330,331-332,335,346$, $347,351-352,366,370,371,430$, 442-444

Warsaw Uprising in $19436,8,9,88,140$, 214, 215, 218, 366, 370, 393, 407, 436

Zbarazh Ghetto 361-362

Gleiwitz (Gliwice) - see concentration camps God

absence of God 222, 242-244

argument with God 90, 112, 444

God's omnipotence in question 46, 47, 94, 111-112, 227, 344, 385, 398

the helpless God 18, 45-48, 458 human beings playing God 282

Goebbels, Joseph - see Nazi leaders and prominent Nazis

good German 32, 58, 68, 74, 86, 127, 142-143, 255, 276, 398, 435

Göring, Hermann - see Nazi leaders and prominent Nazis

Gospels - see Bible/New Testament

Great History - see macrohistory

Gross-Rosen - see concentration camps

grotesque - see comedy

Gypsies - see Roma people 19, 51, 227, 247, $272,315,350,458$

Heaven 18, 45-47, 92-94, 154-155, 378

Hebrew 76, 90, 110, 143, 167, 249, 297, 362, 378, 381, 386, 389, 439

Hebrew Bible - see Bible

Hell 47, 93, 131, 219, 242-244 - also see Devil, Mephisto, Satan

Prince Beelzebub 242

heterodiegetic narrator - see narration

Heydrich, Reinhard - see Nazi leaders and prominent Nazi 
hiding

hiding in an Aryan family $19,22,58,62$, 67-68, 99, 106-107, 151, 157, 208, 212, 215-216, 218-219, 233-235, 256257, 297, 311-312, 355-356, 362-363, 393, 434

hiding in a cave 447

hiding in a cellar 61-62, 329-331, 359

hiding in a forest bunker 351

hiding in a monastery 122,315

hiding in ruins of Warsaw 317

hiding in a shed 169-170, 423-424

hiding in a stable 213

hiding in a well 203

hiding in woods 70

hiding in a zoo 199

history of a place $285-287,446-448$

Hitler, Adolf, Führer - see Nazi leaders and prominent Nazis

Hlinka Guard (Slovak fascists) 66-67, 97, 151$152,155,170,211-212,376-377,384-385$, 401, 404-406

Holocaust kitsch 2, 188, 192, 290

Holocaust Museum in Auschwitz 154-156, 179$181,327,411-412$

Holy Mother 58, 122, 261, 282, 323, 352

Home Army - see Armia Krajowa

Homeric hexameter - see verse

homosexuality - see sex

house theatre 253

humour 24, 25, 31-32, 55, 198, 222, 378, 386-

$387,388-389,427-428$ - also see comedy, irony, satire

bitter laughter 244

black humour 19, 28, 33, 119, 137-138, 170, 251, 259, 378, 388-389, 412, 417, 444

hunger, famine $8,89-90,93,184,243,246$, $258,296,317,415,427,428,451$

identity of Jews $10,12,13,16,25,78,163,198$, $219,223,231,235,243,292,322-323,330-$ 331, 343-344, 346-348, 392, 412, 413, 435-436, 438-440, 448

change of identity $57-58,243,276$

double identity $10,126,163,187,322-323$, 335, 409

European identity 29

false identity - see Aryan papers female identity $13-14$

Jewish vs. Polish identity $177,187-188$, $216,283,335,346-348,359,408-$ 409, 432

lost identity $234,243,292-293,411-412$, 438-440

idyllic life before the war, idyll $50,134,154,162-$

163, 255, 275, 276, 293, 362-363, 377-378, 416, 434435

impersonal narrator - see narration

impossibility of a verbal presentation of the

Shoah - see crisis of literature

individual memory - see memory

informer - see denouncement of Jews

International Red Cross 239, 416, 419, 421

intimate, inner perspective - see narration

irony $47,55,63,108,129,130,198,222,242$,

$332,409,412,417,425,444$ - see also co-

medy, humour, satire

Izbica - see ghettos

Jankiel 351, 352 Jewish character in Mickie-

wicz)

Janowska - see concentration camps

Jeremiah (biblical figure) 321

Jedwabne - see massacre in Jedwabne

Jesus Christ 92-94, 121, 122, 201, 216, 261, 282, $298,312,315,366,431$

Jew - see also stereotypes in presentation of Jews

assimilated Jew $99,157,158,163,241,242$, $256,278,285,342,285,438$

Jewish mother 197-199, 234-235, 322-

323, 351-352

old Jew 23, 24-25, 97, 370, 376-377, 380, 449 - also see retirement home

Orthodox Jew 75, 93, 112, 239, 292-293, $306,344,384,398,428$

wealthy Jew $67-68,339-340,401,449$

White Jew 377

Jewish antisemitism 389

Jewish Bolshevism 97, 386 - also see żydokomuna

Jewish-Christian (non-Jews) relations $6,13,121$ 123, 274-276, 281-283

Jewish Codex (Slovak anti-Jewish laws) 19, 150, 264, 385, 402, 453

Jewish gold 172-174, 238, 311-312, 377, 405

Jewish identity - see identity of Jews 
Jewish jokes 31, 378, 387, 438

Jewish Memorbücher 93

Jewish pets 134, 199, 257, 296, 389

Jewish property

refusing to return Jewish property after the war 275, 285-287

stealing of Jewish property $151,315-316$, 385, 393-394, 401, 404-405

Jewish prostitute 89,126 - also see sex

Jewish resistance - see resistance

Jewish star 100, 133, 134, 158, 263, 296, 330,

343/344, 401, 439

Jewish shtetl 3, 71, 162, 234

Jewish vs. Polish identity - see identity Jewish Welfare Home - see retirement home Jews's Aryan helper 23, 67-68, 111, 133, 151, 166, 215-216, 257, 276, 315, 355, 385, 401, 449-450 - also see hiding in an Aryan family

Judaism 10, 16, 17, 90, 93, 121, 250, 252, 276, 446

Judenrat (Jewish Council)

collaboration of Jewish councils with the

Nazis 94, 130, 191, 192, 453

Elders of the Jews in Theresienstadt 42, 43, 245,416

Judith (biblical figure) 297, 340

justice

demanding fictional/late justice 191

Kaddish 363, 438-440

Kafkaesque manner 19, 258, 297

Kaufering - see concentration camps

Kielce pogrom - see pogrom

kitsch - see Holocaust kitsch

Korczak, Janusz 63, 88, 318, 351, 352, 443, 444

Kresy (Eastern Borderlands) 71, 72, 361, 363

Kurzbach - see concentration camps

labour camps for Jews in Slovakia

Nováky 221-223, 249-250

Sered' 67, 151

laughter - see humour

limit (extreme) situation 5, 20, 68, 71, 74, 115, $123,140,149,155,201,242,243,251,290$, 450

literary reportage - see reportage

martyrdom (literatura martyrologiczna) 5, 28,

33, 183, 200-202 little Czech/Slovak man 19, 22-23, 115, 257 , 296-297, 376-378, 405-406, 450

looking for a new way of writing about the

Shoah - see provocative literary work

Lodz Ghetto - see ghettos

lost childhood - see children

lost identity - see identity of Jews

Lviv Ghetto - see ghettos

Maccabees 366-367

Mach, Alexander (Šaňo) - see prominent Slovak fascists

macrohistory, Great History 222, 276, 279, 293

Majdanek - see concentration camps

male perspective - see narration

March 1968 - see anti-Jewish campaigning in the late 1960 s

martial law in Poland 1981 8, 9, 335, 358, 407, 408, 410

Mary and Martha - see Bible/New Testament

massacre in Jedwabne 26, 307-308

massacre in Khatyn (Belarus) 136-138

massacre in Katyń (Russia) 6, 138

massacres of the Jews by Hungarian nyilasi 227

massacres of the Jews in Ukraine 385

Mauthausen - see concentration camps

Mephisto 22, 191, 340 - also see Devil, Hell, Satan

memory $8,9,10,15,17,21,26,28,29,71,74,81$, 89-90, 93, 104, 121, 136-138, 147-148, $173,176-177,195,213,218,222-223,268$, 293, 299-301, 316, 326-327, 331, 347, 351352, 370-371, 408, 412, 432, 436 collective memory 50, 268, 308, 374, 446 communicative memory, cultural memory $74,307,322-333$

cultural memory $74,188,307,322,454$

family memory $3,13-14,187,279$

individual memory $14,27,61-63,74,162$, 188,412

memory of a place $162,177,188,198,299-$ $300,432,446$

memory of a tree 74

memory transmission 448

Polish-Jewish memory 16

postmemory 3, 77, 231, 322-323

transnational memory 29

Mengele, Josef - see Nazi leaders and prominent Nazis 
menorah 275-276

messianism 31, 323, 367

microhistory 275, 279

Międzyrzec - see ghettos

Mittelbau-Dora - see concentration camps

mixed couples (Aryan boy and Jewish girl) 21, 27, 57, 59, 86, 151, 208, 263-265, 355-356, 397, 400-402

mixed marriage 19, 24, 99, 114, 132, 157, 256, 276, 397, 401

Monowice - see concentration camps

mother - see Jew, Jewish mother

multilingualism 90, 118-119, 226, 378, 428

music in Theresienstadt - see ghettos

myth, mythisation 50, 100-101, 162-163, 215, 219, 283, 301, 314, 323, 332, 398, 448

naive narration - see narration

names of Jews

change of name $84,88,110,132,150,218$, 226, 411

Daniel (symbolic name) 242, 377, 396-397

David (symbolic name) 89, 342-343, 397398

Emanuel (symbolic name) 122

Esther (symbolic name) 355-356, 211-213, 264

Rebecca (symbolic name) 274-276, 297

Ruth (symbolic name) 306

narration

authorial narrator 402

author-narrator 13, 191, 424, 435

autobiographical narrative $7,9,10,11-12$, $17,24,29,32,62,80,90,131,133$, 144-145, 197, 203-204, 222-223, 229, 234-235, 317-318, 348, 363, 409, 426-428, 431

parabiographical narration 131

child's narration, child's point of view 11 , 12, 104, 140-141, 170, 234-235, 274

child's narration \& adult's comments 133134, 388-389, 393

cinematic narration, cinematic approach 108, 454

confession narrative 116-118, 163, 440

diaristic narration 72, 318, 409

ex post perspective $103,119,268$, 447448 - also see hic and nunc perspective female perspective $13,28,197,207-208$, 285-286, 331, 334

first-person narrator 14, 61-64, 133-134, 137, 183-184, 234, 254, 257, 274-276, 287

frame narrative, frame story - see composition

heterodiegetic narrator 101

hic et nunc perspective 46, 119, 290 - also see ex post perspective

impersonal narrator 200, 226, 242

internal \& external narration 73

intimate, inner perspective 21, 141

kaleidoscopic narration 42, 446

male perspective 13

multi-voice structure 267,321

naive narration $311,340,343$

non-diegetic narrator 419

omniscient narrator 10, 58, 158

perpetrator's perspective 22, 32-33, 114115, 254, 289-290, 317-318

personal perspective of narration 11,114 , 355, 386

polyphonic narration $10,29,90$

postmodern narration 30,191-192, 397, 424, 436

third-person narrative 114, 254, 287, 297

victim's perspective 30

Natzweiler-Struthof - see concentration camps

Nazi jargon (phrases) 115, 118, 340

Nazi officers

good Nazi - see good German

sadistic Nazi 28, 121, 167, 255, 339-340

sophisticated Nazi 22, 340

Nazi leaders and prominent Nazis

Eichmann, Adolf 32, 43, 125, 127, 419-420

Goebbels, Joseph 54, 457

Göring, Hermann 54

Heydrich, Reinhardt 18, 103, 104, 105, 257, 355, 356

Himmler, Reinhard 54, 416

Hitler, Adolf, Führer 19, 31, 32, 51, 53-55, 76, 96, 114, 131, 133, 243, 264, 265, $285,287,343,345,401,411,415,431$, 439, 458

Mengele, Josef 54, 119, 157-158, 398

Rahm, Karl 398, 416

Rosenberg, Alfred 166

Wisliceny, Dieter 389, 453 
Noah's Ark - see Bible/Hebrew Bible/ Old Testament

non-diegetic narrator - see narration

normalisation in Czechoslovakia 23-25, 74, 134, 194, 212, 253, 278, 388, 446

Nováky - see labour camps for Jews in Slovakia number (persecuted/prisoner losing their identity) $42,75,86,257$

Nuremberg trials 166,406

Oberlanzendorf - see concentration camps

Old Testament - see Bible

omniscient narrator - see narration

oral history $137,195,424$

oral tradition 448

Orthodox Jew - see Jew

Otwock Ghetto - see ghettos

partisans $20,22,66,67,71,125-127,221,250$, 310-312, 384, 385

perpetrator's perspective - see narration

Pinkas' Synagogue in Prague 21, 103, 158

place - see history of a place

play within a play $173,247,415,444$

pogrom $6,26,161-162,203,209,250,308,374$,

439 - also see massacre

Kielce pogrom 6, 335-336, 393

pogrom in Topolčany 26

Polish-Jewish memory - see memory

Polish-Jewish relations - see relations

Polish-Jewish-German relations - see relations

pop culture 14, 15, 30, 300, 322, 323

post-Holocaust trauma - see survivors or trauma

postmemory - see memory

postmodernism 191

postmodern narration - see narration

posttraumatic stress disorder - see survivors

posttraumatic literature $148,170,227,285-$ 287

postwar "forgetting" of the Jews $58-59,166$, $209,316,374,412-413$

Prague Spring 23, 25, 73, 165, 221, 226, 253 , 274, 388, 426, 449

Prince Beelzebub - see Hell

profanation $7,282-283$

profanation of the Shoah $15,137-138,172,174$, 179-180, 289-290

prollage - see recycling prominent Slovak fascists

Mach, Alexander (Šaňo) 264, 386

Tiso, Jozef 3, 19, 26, 96, 97, 226, 265, 386, 458

Tuka, Vojtech 386

prospective into the future lives - see composition

prostitution - see sex

provocative literary work / controversy 5, 54-55, 119, 137-138, 165, 180-181, 183-184, 191, 212-213, 234, 264-265, 289-290, 299-

300, 307-308, 331-332

public debate 119

new ways of writing $55,119,137-138,252$, 278, 327, 416-417

Pruszków concentration camp - see concentration camps

Pruszków Ghetto - see ghettos

rabbi 296, 297, 340, 453

Ravensbrück - see concentration camps

Rebecca - see names of Jews

reconciliation

German-Polish 59

recycling

history recycling 15, 181

recycling the texts of other authors (prollage) 267, 326-327

Red Army 92, 127, 208, 234

refusing to return Jewish property after the war see Jewish property

relations

Czech-German 54, 103-104, 118, 275-276, 285-287, 296-297, 356, 419-421

Czech-Jewish 18-19, 26, 27, 29, 132-134, 275-276, 355-356, 398

Czech-Jewish-German 287

Czech Jews-Polish Jews 425

Jewish-Christian 5, 17, 92-94, 123, 225226, 235

Polish-German 32, 57-59, 122-123, 176177, 270-272, 317-319, 393, 431, 435, 443-444

Polish-Jewish 4, 6, 7-8, 9, 11, 13-16, 28, 49-51, 57-59, 71, 78, 89-90, 162-163, 176-177, 186, 192, 205, 214-216, 234235, 292-294, 301, 307-308, 322$323,330-331,335-336,362-363$, 392-394, 408-409, 430-432 
Polish-Jewish-German 57-59, 106-108, 335-336

Slovakian-Jewish 17, 19-20, 22-23, 24, 26-28, 66-68, 96-97, 211-212, 384386, 400-402

reportage, literary reportage $127,180,191,332$, $335,346-348,409$

resistance $12,20,35,66,67,71,73,97,142,155$, $166,172,200,249,250,274,276,293,304$, 392, 393, 401, 417, 449-450

Communist resistance 96, 100, 354, 377 Jewish resistance 19, 221, 247, 257, 318 resistance in ghettos and camps $9,28,85$, $140,172,243,419$

retirement home $76-75,147-149,292-293$

returning of Jewish property - see Jewish property

rhymes

nursery rhymes 118

pun / taboo breaking 119

regular 117

Ringelblum Archive 4

ritual murder 301

Roma people - 19, 51, 227, 247, 272, 315, 350, 458

Rosenberg, Alfred - see Nazi leaders and prominent Nazis

Ruth - see names of Jews

Sachsenhausen - see concentration camps Sambor Ghetto - see ghettos

samizdat $6,21,24,73,74,95,102,253,266$, $278,325,362,369$ - also see drugi obieg

Satan 201, 244 - also see Devil, Hell, Mephisto

satire 55, 96-97, 245-247, 278, 442, 444 - also see comedy, humour, irony Menippean satire 321

Schwarzheide - see concentration camps second generation $13,15,27,28,33,62,80-82$, 138, 197, 231, 413, 448

Sefer ha-Zohar 51

September campaign 172, 175, 366, 442

Sered' - see labour camps for Jews in Slovakia sex, sexuality

homosexuality 29,348

homosexuality in ghettos and camps 86 prostitution, prostitute $15,29,49,89,113$, 122, 126, 239

sex in concentration camps 397 sex in Theresienstadt 208, 390, 414

sexual abuse 254, 287, 330, 331, 363, 397

sexual abuse in Auschwitz 147-148

shmaltsovnik - see denouncement of Jews

Shoah as a continuous event 268, 326-327, 457-458 self-renewing circle of evil 268, 282, 326

shtetl - see Jewish shtetl

Slánský trial - see Stalinist antisemitism

Slovak clerofascist jargon (phrases) 97, 212, 386

Slovak National Uprising $20,66,68,95,151$, 249, 303, 311, 400, 443

Slovakian-Jewish relations - see relations

socialist realism $6,21,85,96,129,215,358$

Solidarity movement $61,80,410$

solidarity among prisoners vs. rivalry $85-86$, $141,183,184$

Stalinist antisemitism 20, 23, 134, 226, 356, 390, 426, 457

Slánský trial 20, 24, 41, 133-134, 418, 426

"Zionism" - accusation of the Jews in Stalinist jargon 24, 226, 238, 356, 378, 380

star of David, yellow star identifying the Jews $100,134,257-158,330,343-344$, 401, 439, 450

stealing/theft of Jewish property - see Jewish property

stereotypes in presentation of the Jews 18,26 , $29,215,246,307,308,331$ - also see Jew beautiful Jewess $57-58,126,139,147-148$, 191, 339-340, 447-448

greedy Jew 68, 246, 312, 401

huckster Jew 412

Jew as an animal 424-425

Jew as a businessman 134, 152

Jew as a small mouse 85,402

Jew as an insect 424

Jewish nose 97,356

lecherous Jew 246

stingy Jew 401

Stolpersteine (engraved brass paving stones bearing the names of Jewish victims of the Shoah) 261

suicide $23,88,100,106,111,115,126,127,143$, $145,165,182,196,212,221,238,281,296$, $322,334,366,374,377,378,385,426,443$, $449,450,451$ 
suppressing the memories from the war - see trauma of survivors

survivors $2,8,9,13,14,16,17,25,27,28,29$, $62,137,147-149,151,173,176,198,205$, 209, 229, 231, 235, 237, 241, 245, 262, 275 , 292, 293, 336, 346, 347, 351, 361, 382, 398, 409, 413, 419, 423, 430, 438

post-Holocaust trauma $13,145,147-149$, 231, 275

posttraumatic stress disorder 147-149, 229-231, 411-413

survivors' feelings of guilt - see trauma of survivors

survivors' obligation to commemorate the Shoah 159, 176

surreal scenes - see dream scenes

taboo breaking motifs $55,119,148,172,235$, 289-290, 416

Talmud 51

testimony $9,25,28,43,64,127,162,163,176$, 177, 186, 205, 209, 223, 261, 271, 363, 423, 456 - also see autobiographical narrative, witness

theft of Jewish property - see Jewish property

Theresienstadt - see ghettos

Theresienstadt Gestapo prison (Small Fortress) 253-254

third generation 2, 5, 16, 27, 28, 29, 33, 76-78, 138

third-person narrative - see narration

Tiso, Jozef - see prominent Slovak fascists

Torah, see Bible/Hebrew Bible

Tower of Babel - see Bible/Hebrew Bible/ Old

Testament

transnational memory - see memory

transports

escape from transport $67,140,141,267$, 376,384

transports from Slovakia 19-20, 23, 24, 27, $29,67,95,151,169,195,212,239,260$, 261, 263, 304, 312, 315, 316, 376, 377, $385,386,401,404,405,453,457$

transports from the Czech lands 18, 22, 26, 41-42, 45, 84, 92, 132, 133, 159, 208, 244, 245, 258, 295-297, 342, 355, 356, 388-389, 397, 418, 426, 450

transports from Theresienstadt to death camps $42,45,47,84,99,119,157$,
$245,256,342,397,416,418,420,421$ 426, 428

transports in Poland 63, 107, 143, 172, 183, 184, 190, 200, 289, 373-374, 427, 428, 442

trauma of survivors, traumatisation $11,13,16$, $17,29,43,62-64,88,111,126,143,145$, $148,167,170,187,197,205,212,223,229-$ 231, 234, 237-239, 264, 268, 275, 280, 285-287, 292-293, 312, 329-331, 336, 363, 380-382, 398, 413, 426, 448, 458

missing solidarity and empathy for the traumatised victims 148, 275

phobia of dirt 238, 239

phobia of nudity 239

phobia of showers 380

suppressing the memories from the war 381

survivors' feelings of guilt 209, 237, 279280, 313, 329-331, 398

trauma of returning home $237-239,292-$ 293, 398

traumatic memories, traumatic memory $223,229,448$

traumatic realism 233

Treblinka - see concentration camps

Tuka, Vojtech - see prominent Slovak fascists tzadik 292

Umschlagplatz 63,186-188, 318, 336, 347, 444 undignified death - see death

verse

dactyl 117

free verse $102,105,154,267,374,457$

folk songs \& nursery rhymes 103,118

Homeric hexameter 366

macaronic 118

trochaic 118

victim's perspective - see narration

Warsaw Ghetto - see ghettos, Warsaw Ghetto Warsaw Uprising in 1943 - see ghettos

Warsaw Uprising in 1944 9, 57, 70, 71, 80, 106, $175,186,218,318,329,330,331-332,335$, $350,365,408,423,435$

White Jew - see Jew

Wisliceny, Dieter - see Nazi leaders and prominent Nazis 
witness, eyewitness $4,9,17,22,27,28,41,43$, $77,88,93,107-108,111,113,141,148,163$, $188,191,219-220,222,237,245,250,251$, $258,270,271,290,295,346,350,351,386$, $396,404,418,424,436,447,448$ - also see testimony

women's narrative - see narration

Working Group (illegal Jewish organisation in Slovakia) 386, 453
Yiddish 18, 85, 88, 90, 93, 143, 378, 384, 385, 386,389

Zbarazh Ghetto - see ghettos

Żegota 61, 200

Zionism 24, 47, 104, 106, 110, 121, 384, 385

"Zionism" - see Stalinist antisemitism

żydokomuna 205, 210 
